\title{
Application of Clinical Nursing Pathway for Endoscopic Mucosal Dissection of Tumors
}

\author{
H. XIUQIN*
}

The First Affiliated Hospital of Wenzhou Medical University Department of Gastroenterology, Wenzhou, Zhejiang 325000, China

\author{
Xiuqin: Clinical Nursing in Endoscopic Mucosal Dissection of Tumor
}

This paper investigated the application of clinical nursing pathway in endoscopic mucosal dissection of tumors in patients. Seventy patients with benign gastric tumors were selected as the study subjects. All 70 patients underwent endoscopic mucosal dissection. According to the hospitalization number, they were divided into the study group and the control group, 35 cases in each group. The control group received routine nursing while the study group had the clinical nursing pathway implemented. The postoperative fasting time, hospitalization time and cost, complications and number of relapses were compared between the two groups. The results showed that the fasting time and hospitalization time of the study group were shorter than that of the control group and the treatment cost was lower than that of the control group $(P<0.05)$. The rate of postoperative complication was low in the study group. There was no significant difference in the rate of postoperative recurrence between these two groups. Finally, the clinical nursing pathway was significantly effective in the treatment of tumors with endoscopic mucosal dissection.

Key words: Clinical nursing pathway, tumor, endoscopic mucosal dissection

Endoscopic mucosal dissection is a new treatment for digestive tract diseases ${ }^{[1]}$. It would enable complete removal of the diseased tissue under endoscopy in more patients with early digestive tract tumors to avoid the pain and trauma caused by open surgery ${ }^{[2]}$. Patients in early phase of oncology have a higher success rate, low trauma with higher safety, so they are highly recognized. Compared to traditional surgery, endoscopic mucosal dissection has the advantages of less trauma, quick recovery, low recurrence rate, low treatment cost, shorter hospitalization and complete resection of lesions ${ }^{[3]}$. However, due to its long operation time and high technical difficulty, the incidence of complications such as bleeding and perforation is also high, so it is prone to infection causing anxiety to the patient ${ }^{[4]}$. However, the success of surgery depends not only on the skills of the doctor, but on the effective care by the caregiver system and the close coordination of the 
patient ${ }^{[5]}$. The clinical nursing pathway is a multidisciplinary nursing service and management model composed of medical sciences such as nursing and clinical medicine, nutrition, pharmacy and laboratory medicine ${ }^{[6]}$. The clinical care pathway is the care model during the patient's hospitalization and which is a planned, purposeful, and predictable care work $^{[7]}$. There are two main types of representation, the clinical care pathway is an inpatient care pattern, which is set for a particular type of patient based on a daily standard care plan. It is a combination of each of the patient's diagnostic routine care plans, which can help the caregiver judge the patient's prognosis ${ }^{[8]}$. When the prognosis of a patient changes due to changes in the conditions, the caregiver marks the change in the care path and immediately begins to implement the necessary intervention. The clinical nursing pathway is the nursing mode of the patient during hospitalization. It is aimed at each specific patient group, with time as the horizontal axis, admission guidance, admission diagnosis, examination, medication, treatment, nursing, diet guidance, health education and discharge plan. The ideal nursing method is the vertical axis, which is made into a schedule, which describes and records when and what kind of examination, treatment and care should be done, to what extent the condition is reached and when it can be discharged. With continuous development of endoscopic diagnosis and treatment techniques in the Department of Gastroenterology, endoscopic mucosal dissection has been developed and widely applied. Endoscopic mucosal dissection has the advantages of minimal surgical trauma, high resection rate, less pain and quick recovery. The contraindications of patients are also significantly lower. The clinical nursing pathway is based on a certain disease or surgery, and implements a standardized nursing process that takes patients as the center and allows patients to go from hospital to discharge according to the clinical pathway table. In this investigation, the clinical nursing route is applied to patients with tumors undergoing endoscopic mucosal dissection. Compared to patients who were given routine nursing, the two nursing effects are analyzed and compared and the clinical nursing pathway is studied in patients with tumors who were subjected to endoscopic mucosal dissection. Seventy patients with benign gastric tumors admitted to the First Affiliated Hospital of Wenzhou Medical University were included in the study. Among the 70 patients, 32 were male and 38 were female; the hospitalization expenses ranged from 11557.47 to 33473.3 yuan with the average hospitalization expenses pegged at
$19457.28 \pm 412.85$ yuan. All patients and their families were made aware of the study and had signed an informed consent form, which was approved by the Medical Ethics Committee of the hospital. Patients were informed of possible risks and benefits prior to treatment, volunteered for endoscopic mucosal dissection and signed preoperative informed consent. According to the different nursing methods applied, these 70 patients were divided into two groups, the study group $(n=35)$ and the control group $(n=35)$. The study group had 14 males and 21 females with an age range of 33-75 y and the median age was $52.74 \pm 3.96 \mathrm{y}$. In the control group there were 18 males and 17 females, with an age range of 34-75 y and the median age was $52.85 \pm 4.19$ y. The age data (fig. 1) of the two groups was not statistically different. Inclusion criteria were, patient who were diagnosed as having an early tumor by pathological and endoscopic staining. Patients who met the conditions of endoscopic mucosal dissection. Patients who voluntarily accepted the nursing mode and cooperative to receiving nursing measures, patients of age $18 \mathrm{y}$ and above and are accompanied by family members. Exclusion criteria were, those with liver and kidney dysfunction, severe cardiovascular and cerebrovascular disease and mental depression combined with other serious organ lesions. Patients who have disturbance of consciousness and poor treatment compliance and those who died during the course of this study. The control group was provided with routine nursing methods, and the responsible nurses gave measures such as condition observation, diet, activities, psychology, medication care and health education according to a set routine. The study group were subjected to a clinical care pathway, in which a clinical nursing pathway group of nurses, third-level

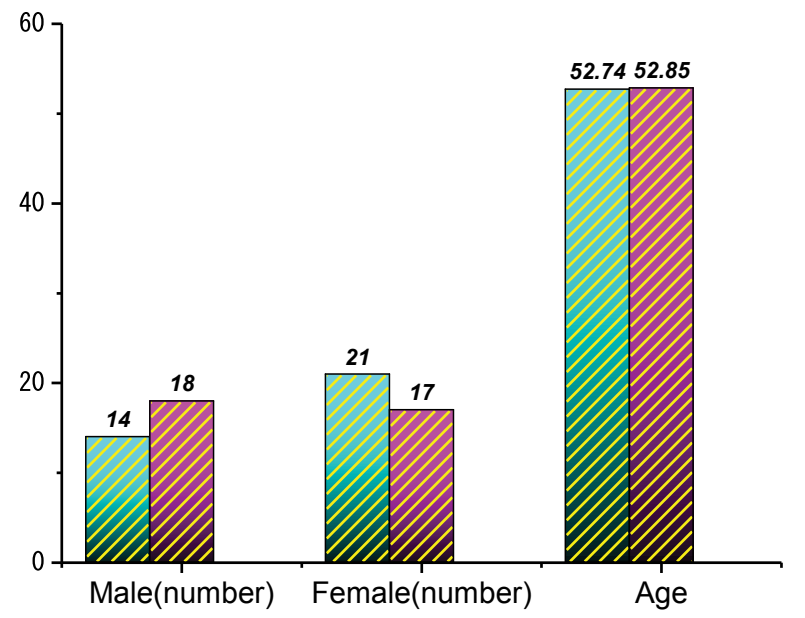

Fig. 1: General information of the two groups Comparison of general information between the research group ( $)$ and the control group (C)

Special Issue 4, 2020 


\begin{tabular}{|c|c|}
\hline Evaluation & $\begin{array}{l}\text { General assessment: current medical history, past history, allergy history } \\
\text { Specialist evaluation: digestive symptoms, defecation, presence or absence of hematemesis }\end{array}$ \\
\hline Examination & Explain notes and tests. \\
\hline Treatment & Symptomatic treatment according to doctor's advice and closely observe changes in the condition \\
\hline Activity & Pay attention to bed rest and reduce body consumption \\
\hline Diet & High-nutrient, high-protein, digestible diet to avoid cold, irritating foods \\
\hline Health education & Health education brochure to introduce ward environment and disease-related knowledge \\
\hline Evaluation & Conduct a specialist assessment to ensure that the patient meets the preoperative indications \\
\hline Examination & Preoperative routine examination; ultrasound gastroscopy to exclude surgical contraindications \\
\hline Treatment & $\begin{array}{l}\text { Follow doctor's advice, anticoagulant treatment, sto } \\
\text { patient to take the intestinal drug correctly, if necess }\end{array}$ \\
\hline ctivity & Pay attention to bed rest and reduce body consumption \\
\hline et & gery, no water for $8 \mathrm{~h}$ \\
\hline Health education & $\begin{array}{l}\text { Explain to the patient the } p \\
\text { introduce successful cases } t\end{array}$ \\
\hline Nur & $\begin{array}{l}\text { Follow the doctor's advice, do a good job of patient identification and endoscopic center nurses to do a } \\
\text { good job; do psychological care, guide patients to avoid emotional stress, and actively cooperate with } \\
\text { treatment. }\end{array}$ \\
\hline Nur & $\begin{array}{l}\text { Closely observe patient's condition and changes in vital signs, do basic nursing and specialist care } \\
\text { (pipeline care, skin care), prevent complications; follow doctor's advice, observe the patient's reaction } \\
\text { after medication. }\end{array}$ \\
\hline $\begin{array}{l}\text { Observation of } \\
\text { complications }\end{array}$ & $\begin{array}{l}\text { Bleeding- patient's vital signs, whether there are symptoms such as hematemesis, melena, monitoring } \\
\text { blood routine. } \\
\text { Perforation- Observe the presence or absence of abdominal pain, abdominal muscle tension, tenderness, } \\
\text { rebound tenderness, blood pressure drop, increased pulse, pale, immediately notify the doctor, if } \\
\text { necessary, surgical treatment } \\
\text { Infection- closely monitor body temperature, follow doctor's advice, and disinfection } \\
\text { Anxiety- observe if patient has irritability, inattention, muscle tension and other anxiety, keeping the } \\
\text { ward clean, quiet and comfortable. Strengthen communication, provide disease-related knowledge to } \\
\text { patients, psychological counseling to improve compliance with treatment and care }\end{array}$ \\
\hline Health education & Assist patients in oral care during fasting; dietary guidance during recovery period. \\
\hline Health education & $\begin{array}{l}\text { Instruct patients to work and rest; eat small quantities of light, easy to digest food, take medication } \\
\text { according to doctor's advice, regular review; abdominal pain, bloating, hematemesis and melena and } \\
\text { other timely medical treatment. }\end{array}$ \\
\hline
\end{tabular}

nurses and nurses with experience of $5 \mathrm{y}$ was established. The members of this group analyzed and discussed the data by reviewing the literature, guidelines and other materials, summed up the past experiences and developed an evidence-based and operable clinical nursing path table as per specifications (Table 1). Responsible nurses perform surgical care on patients according to the clinical pathway table. Nursing measures were implemented in 5 stages on the day of admission, preoperative, on the day of surgery, 1-5 d after surgery and on the day of discharge. The hospitalization time, treatment cost and postoperative fasting time of the 2 groups were observed and compared. The recurrence and complications of the 2 groups were compared with the nursing satisfaction. Using SPSS22.0 statistical software, the count data was expressed in the form of $\mathrm{n}(\%)$, the data was analyzed using $\chi^{2}$, the measurement data was expressed in the form of mean \pm standard deviation, $t$ test was used and $\mathrm{P}<0.05$ was used as the difference. The complication rate of the study group was $5.71 \%$, which was significantly lower than that of the control group
( $22.86 \%, \mathrm{P}<0.05$, Table 2$)$. The results showed that the clinical care pathway helped to reduce the incidence of postoperative complications. Endoscopic mucosal dissection is a minimally invasive technique that has emerged with the development of endoscopic diagnosis and treatment techniques as well as the development of endoscopic accessories ${ }^{[9]}$. The success of the operation is not only related to the experience and technology of the physician, but to the nursing staff and effective care The implementation of the clinical nursing pathway can clarify the job responsibilities of the nursing staff, so that they can comprehensively and accurately observe the disease, detect the oozing and perforation early and take corresponding treatment measures in time, so that the quality of care can be comprehensively improved and reduced the incidence of complications. The hospitalization time and hospitalization expenses of the study group were significantly lower than that of the control group $(\mathrm{P}<0.05)$ as shown in Table 3 . It can be seen that the clinical nursing pathway could shorten the average hospitalization time and reduce hospitalization cost. Because of the implementation of the standardized 
process, the clinical nursing path enabled patients to ihave nursing care as long as they were admitted to the hospital according to a complete and comprehensive nursing plan $^{[10]}$. The standard hospitalization time are defined by the treatment costs, examination fees and medicine fees and the doctors and nursing staff are reduced, which allowed the patient's medical treatment time and treatment costs to be well controlled, thereby shortening the average hospitalization stay and reducing medical expenses. The clinical nursing pathway is used for patients undergoing endoscopic mucosal dissection. The patients could clearly understand his or her condition which is being treated, identify the treatment goals and actively cooperate with the work to improve his self-care ability and treatment compliance. The nursing satisfaction of the study group was $97.14 \%$, and the nursing satisfaction of the control group was $82.86 \%$, which was statistically significant (Table 4). The core of the clinical nursing path management is the diagnosis and treatment process. Standardized management is aimed to ensure that patients get the right diagnosis and treatment services at the right time and in the right place, so that the overall care of the responsibility system can be better implemented and the seamless management of the patient's perioperative period can be implemented to form a standardized nursing model. In order to standardize the management of the hospital, the nursing staff can actively work along the path content and illustrations to achieve qualification, quantification, timing, and personalization, avoiding omissions in the handover or different personal work habits and care errors, which ensure the continuity of overall care, significantly improve the quality of care and work efficiency. The clinical nursing path was implemented for patients undergoing endoscopic mucosal dissection. Responsible nurses comprehensively intervened from condition observation, psychological care, treatment and health education, which improved the relationship between nurses and patients, the quality of care, and increased patient care satisfaction. Endoscopic mucosal dissection is a new technique for the treatment of tumors, requiring higher levels of clinical care during treatment and rehabilitation. Endoscopic mucosal dissection does not cause serious damage to the patient and can also guarantee the same therapeutic effect like traditional surgery. More and more tumor cases are treated with endoscopic mucosal dissection. In the treatment of tumor patients with endoscopic mucosal dissection, combined with effective nursing intervention has a positive effect on reducing the patient's symptoms, postoperative complications, and the nursing intervention, which is good for all aspects of the postoperative period. Control, which plays an active role in promoting postoperative rehabilitation and assisting in improving the postoperative period of the patient and the patient's nursing intervention involves a comprehensive state of physiology and psychology. Therefore, it is clinically highly effective. The survey found that most of patients who underwent endoscopic mucosal dissection before the treatment of minimally invasive surgery, limiting the patient's intraoperative

TABLE 2: COMPARATIVE ANALYSIS OF COMPLICATIONS

\begin{tabular}{lccccc}
\hline Group & Number of cases & Bleeding & Perforation & Abdominal pain, bloating & Incidence rate \\
\hline Research group & 35 & $1(2.86)$ & 0 & $1(2.86)$ & $2(5.71)$ \\
Control group & 35 & $3(8.57)$ & $1(2.86)$ & $4(11.43)$ & $8(22.86)$ \\
$\chi^{2}$ value & & & & 4.200 \\
P value & & & $<0.05$ \\
\hline
\end{tabular}

TABLE 3: COMPARISON OF HOSPITALIZATION STAY, HOSPITALIZATION EXPENSES AND POSTOPERATIVE FASTING TIME BETWEEN THE TWO GROUPS

\begin{tabular}{lccc}
\hline Group & Hospital stay $(\mathrm{d})$ & Hospital costs (Thousand yuan) & Fasting time $(\mathrm{h})$ \\
\hline Research group $(n=35)$ & $9.23 \pm 2.87$ & 8.569 & $24.58 \pm 3.27$ \\
Control group $(n=35)$ & $12.85 \pm 3.96$ & 12.587 & $40.63 \pm 5.25$ \\
$t$ value & 4.379 & 129.686 & 14.969 \\
$P$ value & $<0.05$ & $<0.05$ & $<0.05$ \\
\hline
\end{tabular}

TABLE 4: NURSING SATISFACTION WAS COMPARED BETWEEN THE TWO GROUPS

\begin{tabular}{|c|c|c|c|c|c|}
\hline Group & Number of cases & Very satisfied & Satisfied & Not satisfied & Level of Satisfaction \\
\hline Research group & 35 & $20(57.14)$ & $14(40.00)$ & $1(2.86)$ & $34(97.14)$ \\
\hline Control group & 35 & $15(42.86)$ & $14(40.00)$ & $6(17.14)$ & $29(82.86)$ \\
\hline$\chi^{2}$ value & & & & & 5.0806 \\
\hline$P$ value & & & & & $<0.05$ \\
\hline
\end{tabular}


fit, but reduced the success rate of surgery due to bleeding and perforation during surgery. Symptoms are also a common phenomenon. If not controlled in time, it is very likely that the endoscopic mucosal dissection treatment could not be performed but patients have to undergo traditional surgical treatment. Clinical studies have found that the routine nursing mode cannot meet the needs of endoscopic submucosal dissection treatment. The effect is not ideal and the control of complications is not good, so the clinic needs to change the nursing mode, the more perfect and rigorous nursing procedures is the premise to ensure effective treatment with endoscopic mucosal dissection. The clinical nursing path is a comprehensive new nursing model that runs through multiple disciplines. Through the individual responsibility of the doctors and nursing staff in each department, individualization of patient is combined with a nursing workload scale under the guidance of the clinical nursing pathway. The nursing model not only enhances the ideal of evidence-based medicine but implements the normal and planned implementation of nursing work. The nursing staff can know exactly what time to do and how to do it. The nursing mode is more scientific and effective in coordinating the whole treatment preparation and postoperative rehabilitation measures. In the nursing process, it is effectively matched with the clinical treatment, assisting and promoting each other and achieving the expected effect, so have a positive clinical effect in terms of improving work efficiency and effect. At the same time, the research on the nursing effect of clinical nursing pathways in patients with diseases is more common and most departments and diseases are getting involved, which also determine the advantages of its effects. The patients are assisted in treatment, so the postoperative treatment time is shortened. The effect is obvious in reducing complications in some related clinical studies on the nursing effect of patients undergoing endoscopic mucosal dissection. Although reports on the effectiveness on the hospitalization time for patients are still relatively scarce, there is a still big difference in the impact on treatment costs and postoperative fasting time, so the space for exploration in this area is still large. The results showed that the surgery of the patient and the disease before surgery could be improved if the patient and the family were aware of the operation, which enhances the confidence of endoscopic mucosal dissection, preoperative fasting to ensure smooth operation Intraoperative, cooperation with the physician to prevent complications of bleeding and perforation, closely monitor the patient's vital signs after surgery, observe the changes in patient's condition, take timely and response measures, pay attention to the patient's postoperative diet, according to the patient's postoperative situation follow doctor's advice to help patients recover from their activities at different times after surgery. Follow up after discharge from the hospital, so that patients can review on time, reflecting the continuity of care. Therefore, it is extremely necessary to use the clinical nursing path to develop professional care content according to the patient's specific situation. At the same time, in the process of formulating the clinical nursing pathway table, the doctors and other department staff are fully and meticulously communicated. In addition, effectively refining the nursing measures, the nursing staff also have a prior understanding and mastery of the entire nursing process and enhance the nursing staff. Learning mastery of relevant nursing content, and the systematic characteristics of the implementation are more significant. When the implementation process is more fluent, the details are more precise and the patient's acceptance and recognition of care are relatively higher, so the effect is better. The clinical nursing pathway is a comprehensive and interdisciplinary medical nursing model. It is also a tool scale, which fully reflects the concept of evidence-based medicine, adheres to the principle of continuous improvement of quality management, standardize hospital management, and improve the quality of care. The clinical nursing path has many advantages in improving the degree of cooperation between the nursing staff and the treating physician. Through the formulation and implementation of the nursing measures, the degree of cooperation between the two is improved, thereby ensuring the smoothness and effectiveness of the implementation of nursing measures.

In this study, patients with tumors undergoing endoscopic mucosal dissection were provided with clinical care pathway intervention. The length of hospital stays, treatment cost, and postoperative fasting time were significantly less than those in the control group. The number of complications in the study group was lower than that in the control group. It can be seen that the nursing model under the guidance of the clinical nursing path has a better clinical treatment effect, which can effectively reduce the occurrence of postoperative complications and has obvious advantages in improving patient satisfaction. In summary, when patients with benign gastric tumors underwent endoscopic mucosal dissection, application of the clinical nursing pathways 
have obvious effects, which is worthy of further clinical application and promotion.

\section{REFERENCES}

1. Boora P, Rathee M, Bhoria M. Effect of Platelet Rich Fibrin (PRF) on Peri-implant Soft Tissue and Crestal Bone in OneStage Implant Placement: A Randomized Controlled Trial. J Clin Diagn Res 2015;9(4):18-21.

2. Chang JG, Li HW. The Study on Key Factors Influencing Time Delay of Public Construction Project. Adv Mate Res 2014;912-914: 1838-41.

3. EÖ, Alaaddinoğlu EE. The effect of platelet-rich fibrin on implant stability. Int $\mathrm{J}$ Oral Maxillofac Implants 2015;30(3):578-82.

4. Gaži P, Pietrzak K, Tessaro S. The Exact PRF Security of Truncation: Tight Bounds for Keyed Sponges and Truncated CBC. Cryptol Conference 2015;368-87.

5. Hiremath H, Saikalyan S, Kulkarni SS, Hiremath V. Secondgeneration platelet concentrate (PRF) as a pulpotomy medicament in a permanent molar with pulpitis: a case report. Int Endod J 2015;45(1):105-12.

6. Julia H, Thomas S, Markus S, Markus S. The effect of PRF (platelet-rich fibrin) inserted with a split-flap technique on soft tissue thickening and initial marginal bone loss around implants: results of a randomized, controlled clinical trial. Int J Implant Dent 2016;2(1):1-10.

7. Kobayashi E, Flückiger L, Fujiokakobayashi M, Sawada K, Sculean A, Schaller B, et al. Comparative release of growth factors from PRP, PRF, and advanced-PRF. Clinical Oral Investigations 2016;20(9):2353-60.

8. Kumar PG, Kumar GS, Kumar SCV. Platelet Rich Fibrin (PRF) in Regeneration of Intrabony Defects- A Randomized Controlled Trial. J Periodontol 2017;88(11):1-14.

9. List E, Nandi M. Revisiting Full-PRF-Secure PMAC and Using It for Beyond-Birthday Authenticated Encryption. Cryptographers' Track at the RSA Conference 2017;258-74.

10. Moy RL, Chen LS, Kao LJ. Multiple Linear Regression. World Scientific Book Chapters 2016;93(12):364-8.

This is an open access article distributed under the terms of the Creative Commons Attribution-NonCommercial-ShareAlike 3.0 License, which allows others to remix, tweak, and build upon the work non-commercially, as long as the author is credited and the new creations are licensed under the identical terms

This article was originally published in a special issue: Special issue on "Animal Models \& Experimental Medicine"

Indian J Pharm Sci 2020:82(2)spl issue4;92-97 